\title{
London Dispersion Corrections to Density Functional Theory for Transition Metals Based on Fitting to Experimental Temperature- Programmed Desorption of Benzene Monolayers
}

\author{
Hao Yang, Tao Cheng,* and William A. Goddard, III* \\ Cite This: J. Phys. Chem. Lett. 2021, 12, 73-79 \\ Read Online
}

ACCESS 1

Llll Metrics \& More

回 Article Recommendations

Supporting Information

ABSTRACT: Standard implementations of generalized gradient approximation (GGA)based density functional theory (DFT) describe well strongly bound molecules and solids but fail to describe long-range London dispersion or van der Waals (vdW) attraction interactions that are important in molecular crystals and two-dimensional solids. To provide accurate values for the vdW distance and energies for the metals $\mathrm{Cu}, \mathrm{Ag}, \mathrm{Au}, \mathrm{Ni}$, $\mathrm{Pd}$, and $\mathrm{Pt}$, we determined empirical vdW corrections to Perdew, Burke, and Ernzerhof (PBE) DFT by fitting the experimental adsorption enthalpies measured by temperatureprogrammed desorption (TPD) from benzene monolayers by Campbell and co-workers (J. Phys. Chem. C 2016, 120, 25161-25172). Benzene physisorbed to these metals without chemical reaction; therefore, we consider the bonding to be vdW. We use the low gradient form for the vdW corrections, $E_{\mathrm{vdW}-\mathrm{LG}}=-C_{6 \mathrm{LG}} /\left[R^{6}+R_{\mathrm{vdwLG}}{ }^{6}\right]$ with just two parameters per atom $\left(C_{6 \mathrm{LG}}\right.$ and $\left.R_{\mathrm{vdwLG}}\right)$. This LG form leads to negligible changes in bond distances and angles, so adjusting the parameters should not sacrifice accuracy for the bonding interactions. We demonstrate that the parameters fitted to benzene also describe well the physisorption enthalpies for other hydrocarbons (naphthalene, cyclohexane, methane, ethane, and propane) on Pt. We also report low gradient vdW correction parameters for the noble gases that fit the equilibrium lattice parameter and heat of vaporization of the crystals.

$\mathrm{t}$ is well-known that GGA-based DFT functionals, such as PBE developed by Perdew, Burke, and Ernzerhof, fail to
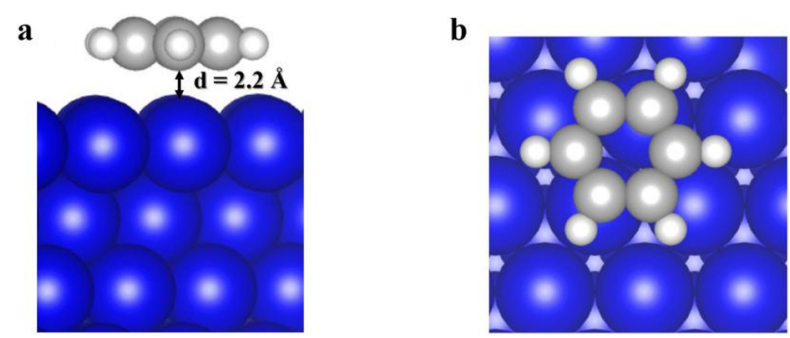

Figure 1. Optimized physisorbed structure of benzene (at the coverage of $0.111 \mathrm{ML}$ ) on the $\mathrm{Pt}(111)$ surface. (a) Side view. The minimized structure has the benzene plane midpoint 2.20 Å above the midplane of the top Pt layer. (b) Top view. The carbon atoms are in bridging positions, while the $\mathrm{H}$ atoms are nearly on-top. The colors are $\mathrm{Pt}$ in blue, $\mathrm{C}$ in gray, and $\mathrm{H}$ in white.

describe the long-range van der Waals (vdW) attraction interactions (London dispersion). This is because vdW attraction arises from instantaneous electron correlations between electrons on adjacent atoms, which is not captured by the average electron density. ${ }^{2}$ More rigorous functionals with London dispersion included are under development, ${ }^{3-5}$ but they are not yet ready for transition metals. As we know,
Grimme's DFT-D methods are quite popular and have been widely validated in many molecular and periodic systems. For instance, for nonmetallic systems that form molecular crystals, a practical solution was developed by Grimme with parameters optimized by Becke-Johnson. ${ }^{6,7}$ Indeed, for nonmetallic systems involving first- and second-row elements (the $C$ and $\mathrm{Si}$ rows), this $\mathrm{D} 3$ correction does quite well in reproducing the experimental equation of state of molecular crystals up to $\sim 100$ $\mathrm{GPa}$ (after including the phonons to calculate zero-point energy and temperature corrections to the enthalpy). ${ }^{8}$

However, metallic systems do not exhibit molecular crystals, making it difficult to estimate accurate vdW corrections. The target of this Letter is to accurately describe the organic-metal interactions important for electrochemical reactions, such as hydrogen evolution reaction $(\mathrm{Pt}),{ }^{9}$ oxygen reduction reaction $(\mathrm{Pt}),{ }^{18}$ and $\mathrm{CO}_{2}$ reduction $(\mathrm{Au}, \mathrm{Cu}$, and $\mathrm{Ag}),{ }^{11,12}$ when $0.1 \mathrm{eV}$ energy difference makes large deviation in prediction $^{13}$ as measured using temperature-programmed desorption (TPD) by Campbell and co-workers, ${ }^{1,14}$ who measured the accurate

Received: October 14, 2020

Accepted: December 3, 2020 

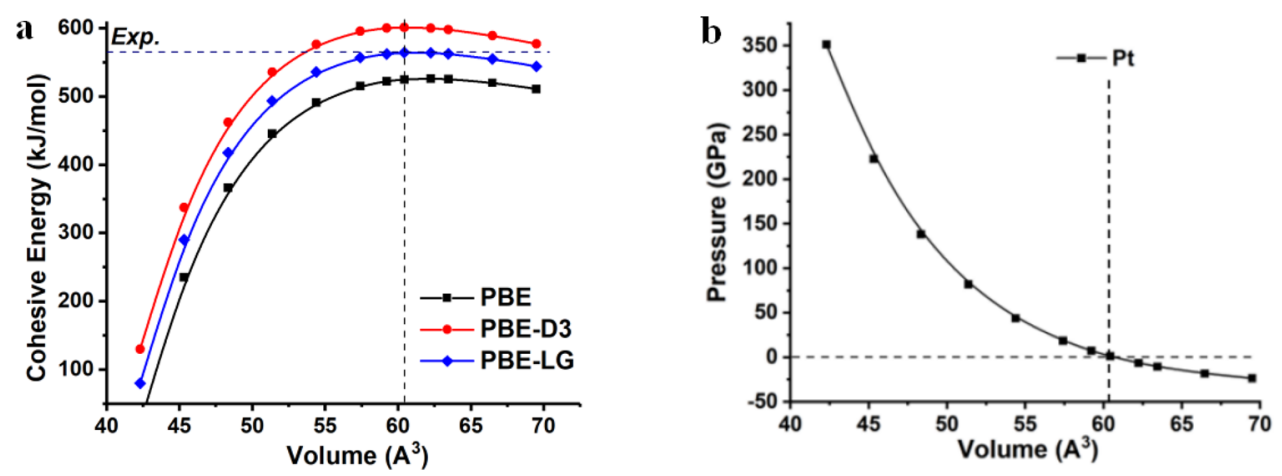

Figure 2. (a) Comparison of the calculated cohesive energy of Pt crystal for various DFT methods compared to the Campbell experiments. (b) Predicted and experimental equation of state for Pt crystal at $0 \mathrm{~K}$. Here we used the minimized structure from the DFT calculations (with or without $\mathrm{vdW}$ corrections) and the calculated vibrational energies to obtain the energy and pressure at the experimental temperature.

Table 1. Adsorption Energies from DFT Calculations to a Single Layer of Physisorbed Benzene Including vdW Corrections Compared to Experiment ${ }^{a}$

\begin{tabular}{|c|c|c|c|c|c|c|}
\hline & \multicolumn{3}{|c|}{ adsorption of benzene on metal surface $\left(\mathrm{kJ} \cdot \mathrm{mol}^{-1}\right)$} & \multirow[b]{2}{*}{$R_{\mathrm{vdwLG}}(\AA)$} & \multirow[b]{2}{*}{$\mathrm{C}_{6 \mathrm{LG}}(\mathrm{eV})$} & \multirow[b]{2}{*}{$S$ (cross-term factor for organic) } \\
\hline & $E_{\mathrm{PBE}-\mathrm{D} 3}$ & $E_{\mathrm{PBE}-\mathrm{LG}}$ & $\operatorname{exptl}^{1,9}$ & & & \\
\hline $\mathrm{Pt}(3 \times 3)$ & -156.60 & -164.02 & $-164(T=300 \mathrm{~K})$ & 6.492 & 2520.5 & 0.5919 \\
\hline $\mathrm{Cu}(3 \times 3)$ & -96.72 & -67.98 & $-68(T=225 \mathrm{~K})$ & 4.594 & 455 & 3.2500 \\
\hline $\mathrm{Ag}(3 \times 3)$ & -80.72 & -62.66 & $-63(T=210 \mathrm{~K})$ & 4.992 & 410 & 0.2680 \\
\hline $\mathrm{Au}(3 \times 3)$ & -87.24 & -72.12 & $-72(T=230 \mathrm{~K})$ & 6.898 & 12257 & 0.1872 \\
\hline $\mathrm{Ni}(3 \times 3)$ & -184.39 & -166.90 & $-167(T=90 \mathrm{~K})$ & 4.492 & 99.49 & -0.3462 \\
\hline $\operatorname{Pd}(3 \times 3)$ & -173.70 & -165.67 & $-166.5(T=300 \mathrm{~K})^{b}$ & 4.996 & 356 & -0.2089 \\
\hline
\end{tabular}

${ }^{a}$ In this table, the metal vdW parameters are for $\mathrm{M}-\mathrm{M}$, but we need to scale $C_{6}$ by the factor $S$ before applying the combination rule for $\mathrm{Pt}-\mathrm{C}$ and $\mathrm{Pt}-\mathrm{H}$. Here, we combined the minimized structure from the DFT calculations (with vdW corrections) with calculated vibrational energies to obtain the binding enthalpy at the experimental temperature. Then we determined the two-body $R_{\mathrm{vdw}}$ and $C_{6}$ parameters to reproduce the experimental binding enthalpy. ${ }^{b}$ The binding of benzene to $\mathrm{Pd}$ used the average for the data of $\mathrm{Ni}$ and $\mathrm{Pt}$ because no experimental data is available (suggested by Charles T. Campbell, private communication).
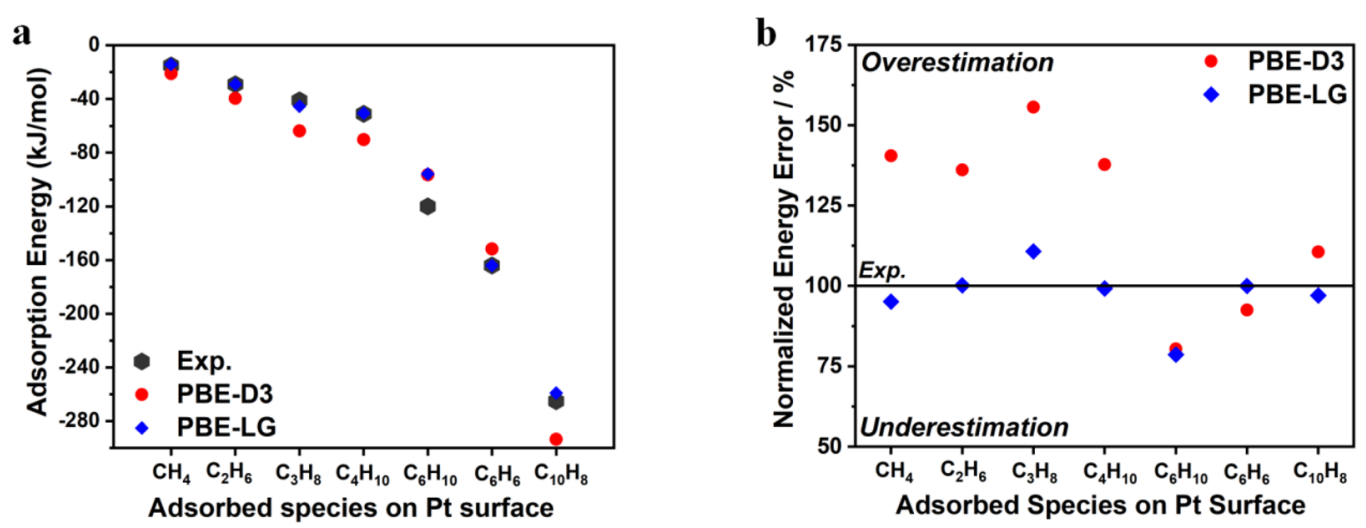

Figure 3. PBE-LG predicted adsorption energy for 6 hydrocarbons and benzene (training set) on Pt(111) surface. (a) Comparisons of adsorption energies with experiment and (b) normalized relative error for each method. Here we have used the minimized structure from the DFT calculations (with or without $\mathrm{vdW}$ corrections) and the calculated vibrational energies to obtain the energy and pressure at the experimental temperature of $300 \mathrm{~K}$.

physisorption energetics for the first monolayer of benzene on these metals.

We also determined the vdW parameters for the noble gases based on fitting the crystal structure, heat of vaporization, and equation of state.

We will focus on two sets of atoms in this work:

- Transition metals of columns 10 and 11 on the periodic table: $\mathrm{Ni}, \mathrm{Pd}, \mathrm{Pt}, \mathrm{Cu}, \mathrm{Ag}$, and $\mathrm{Au}$, where we fit the LG form

$$
E_{i k \text {-dispersion }}=-\sum_{i k, i<k}^{\mathrm{N}} \frac{C_{6 \mathrm{LG}-i k}}{R_{0 i k}^{6}+R_{\mathrm{vdwLG}-i k}^{6}}
$$

to the experimental temperature-programmed desorption (TPD) data of physisorbed benzene.

Here $C_{6 \mathrm{LG}-\mathrm{ik}}$ and $R_{\mathrm{vdwLG}-\mathrm{ik}}$ are optimized to fit binding of physisorbed molecules for atoms $i$ and $k$ and $R_{i k}$ is the variable distance between atoms $i$ and $k$.

- The noble gas crystals ( $\mathrm{He}, \mathrm{Ne}, \mathrm{Ar}, \mathrm{Kr}, \mathrm{Xe}$, and $\mathrm{Rn}$ ), where we fit the LG van der Waals corrections to the experimental lattice parameters, the heat of vaporization, and equation of state.

Physisorbed Enthalpies for Columns 10 and 11 of the Periodic Table. We will discuss the $\mathrm{Pt}$ case in detail followed by a summary of results for the other metals. 

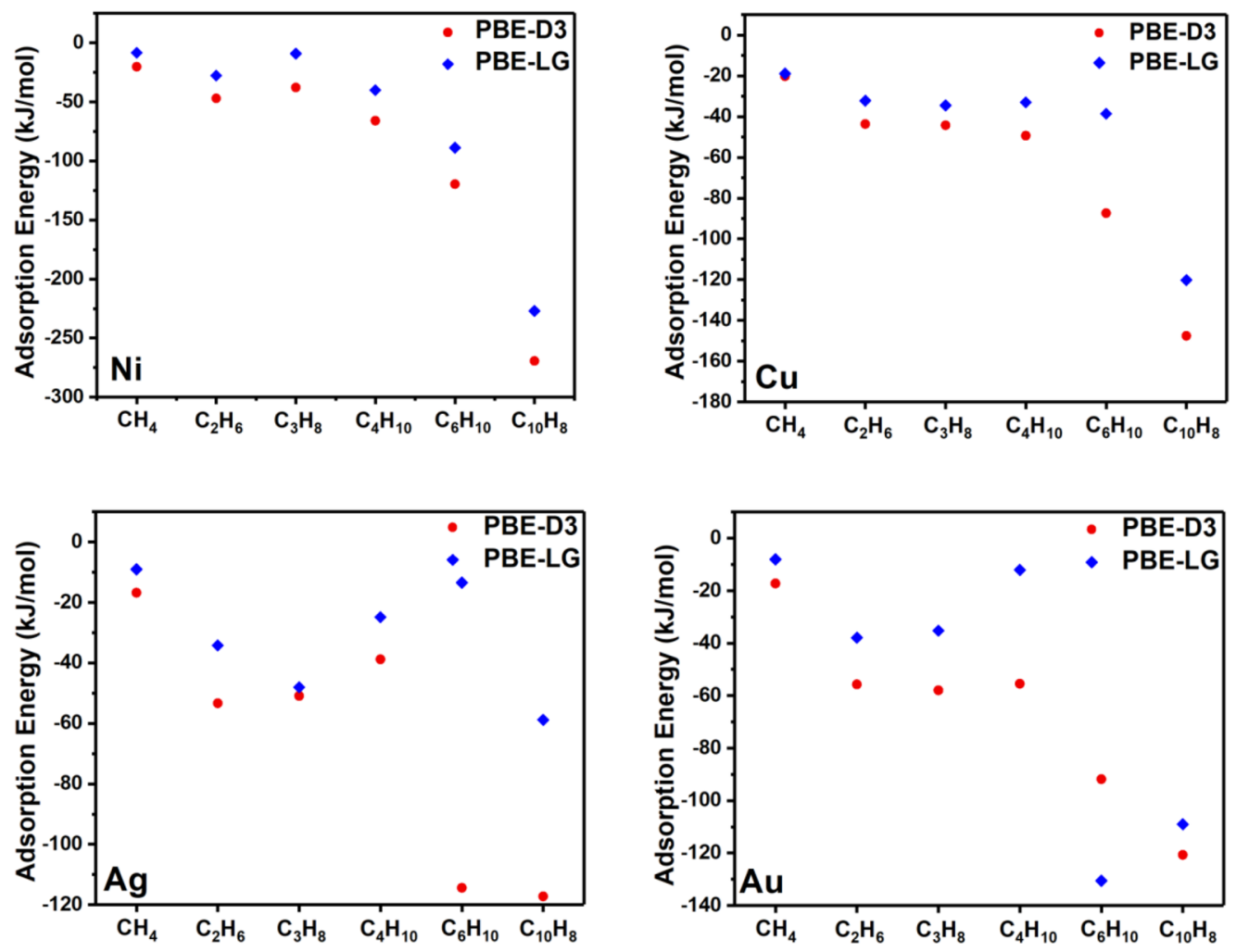

Figure 4. Predicted physisorption enthalpies of hydrocarbons on metals $(\mathrm{Ni}, \mathrm{Cu}, \mathrm{Ag}$, and $\mathrm{Au})$ at the same temperature as in $\mathrm{Table} 1$.

Table 2. Cohesive Energy from Various DFT Methods Compared to Experiment (Extrapolated to $0 \mathrm{~K})^{a}$

\begin{tabular}{|c|c|c|c|c|c|}
\hline & \multicolumn{3}{|c|}{ cohesive energy $\left(\mathrm{kJ} \cdot \mathrm{mol}^{-1}\right)$} & \multirow[b]{2}{*}{$R_{\mathrm{vdwLG}}(\AA)$} & \multirow[b]{2}{*}{$C_{6 \mathrm{LG}}(\mathrm{eV})$} \\
\hline & $E_{\text {PBE-D3 }}$ & $E_{\text {PBE-LG }}$ & $\operatorname{exptl}^{15}$ & & \\
\hline $\mathrm{Pt}$ & 602.3 & 566.2 & 563.5 & 6.492 & 4258 \\
\hline $\mathrm{Cu}$ & 384.2 & 337.3 & 336.7 & 4.594 & 140 \\
\hline $\mathrm{Ag}$ & 290.6 & 285.0 & 284.6 & 4.992 & 1530 \\
\hline $\mathrm{Au}$ & 342.0 & 367.8 & 367.6 & 6.898 & 12257 \\
\hline $\mathrm{Ni}$ & 497.7 & 428.4 & 428.4 & 4.492 & -287.41 \\
\hline $\mathrm{Pd}$ & 501.1 & 375.3 & 375.3 & 4.996 & -1704 \\
\hline
\end{tabular}

${ }^{a}$ Here we used the minimized structure from the DFT calculations (with vdW corrections) and the calculated vibrational energies to obtain the energy and pressure at the experimental temperature.

Physisorbed Benzene. The TPD experiments find that the first layer of physisorbed benzene packs with one molecule per $3 \times 3$ cell on $\mathrm{Pt}(111)$, leading to $0.111 \mathrm{ML}$ coverage (Figure S1). ${ }^{1,14}$ Experiments find that desorption of this layer at $300 \mathrm{~K}$ requires $164 \mathrm{~kJ} / \mathrm{mol}$.

PBE-D3 leads to the optimized geometry shown in Figure 1, which has the benzene plane midpoint $2.21 \AA$ above the midplane of the top Pt layer, with a DFT binding energy of $-156.6 \mathrm{~kJ} / \mathrm{mol}$.

To compare with the free energy as measured experimentally, we calculate the phonons of the benzene layer and the top layer of $\mathrm{Pt}$ and correct for the zero-point energy (ZPE) and the specific heat to obtain the enthalpy at $300 \mathrm{~K}$ (Figure 2). We also calculate the interaction energy between the benzene (within this layer) and Pt to obtain the energy to desorb benzene molecules from the surface (see more details in Table S2).

We want to modify the vdW interactions to fit the experimental binding enthalpy, but we assume that the equilibrium distance of the benzene plane to the Pt surface of
2.21 Å from PBE-D3 may be accurate. Thus, we fitted the well depth to adjust the binding energy, while keeping the distance unchanged. Then, using our new parameters we make any further adjustments needed. The new parameters, $C_{6 \mathrm{LG}-\mathrm{PtPt}}$ and $R_{\mathrm{vdwLG}-\mathrm{PtPt}}$ are listed in Table 1 . Our new binding energy of benzene on $\mathrm{Pt}$ of $-164.02 \mathrm{~kJ} / \mathrm{mol}$ reproduces the experimental results of $-164 \mathrm{~kJ} / \mathrm{mol}$ at $300 \mathrm{~K}$. This changes the Pt surface benzene physisorption distance slightly to $2.20 \AA$.

Predicted Physisorption of Six Other Organics to Pt(111). Having optimized $C_{6 \mathrm{LG}}$ and $R_{\mathrm{vdwLG}}$ for benzene, we now predict the binding energy for 6 other organics measured by Campbell. Figure 3 shows that this leads to a reasonable agreement with the experiment, indicating that PBE-LG can be used for predicting binding for other physisorbed molecules.

We also predicted the adsorption behavior of these six hydrocarbons for $\mathrm{Cu}, \mathrm{Ag}, \mathrm{Au}, \mathrm{Pd}$, and $\mathrm{Ni}$, with results as in Figure 4 and Table S3.

Bulk Cohesive Energies. We had hoped that the LG parameters for benzene physisorbed on $\mathrm{Pt}$ might also lead to the correct cohesive energy of Pt. Thus, we calculated the cohesive energy of pure bulk $\mathrm{Pt}$ ( $\mathrm{fcc}$ ) using the LG parameters (Table 2). Using the same $R_{\mathrm{vdwLG}}$ as for binding benzene to the surface we find that $C_{6 \mathrm{LG}-\mathrm{PtPt}}=4258 \mathrm{eV}$ is required for the bulk cohesive energy, whereas $C_{6 \mathrm{LG}-\mathrm{PtPt}}=2520.5 \mathrm{eV}$ was required for the physisorbed benzene, which is reduced by a factor of 0.5919 . This scaling factor is different for each metal element. Thus, we cannot use the same $C_{6}$ for bulk $\mathrm{Pt}$ as for physisorption on Pt surfaces.

$\mathrm{Cu}, \mathrm{Ag}$, and $\mathrm{Au}$. Figure 5 shows the calculated cohesive energy of pure fcc $\mathrm{Cu}, \mathrm{Ag}$, and Au crystal using optimized LG parameters, which are in good agreement with the experiment.

$\mathrm{Ni}$ and $\mathrm{Pd}$ Cases. The optimal LG parameters for Ni and Pd are summarized in Table 1. As discussed in the Supporting Information, the calculation of $\mathrm{Ni}$ atom in VASP assumes that each of the five $3 \mathrm{~d}$ orbitals has $8 / 5$ electrons, which leads to a 

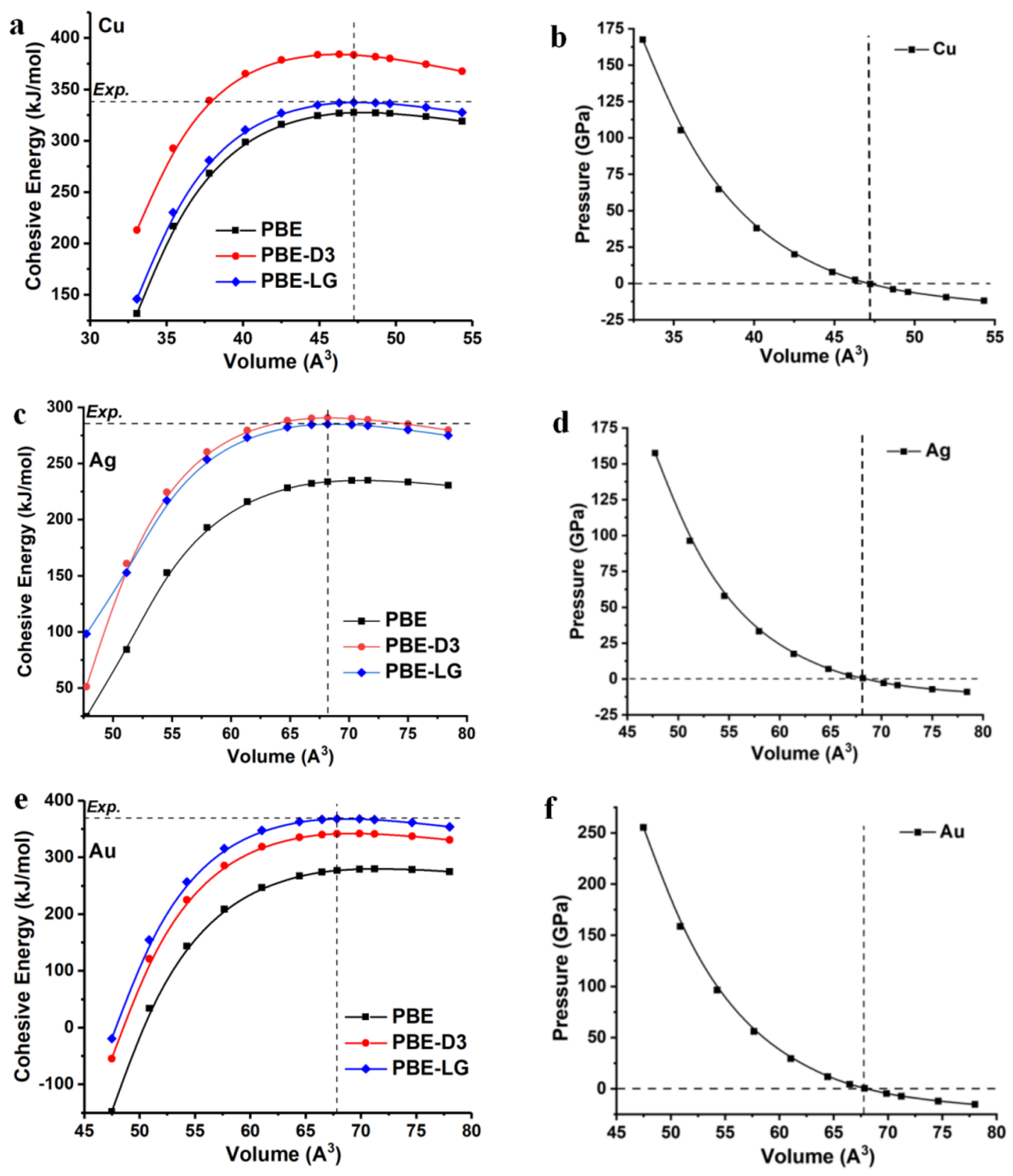

Figure 5. Calculated cohesive energy for crystals using PBE, PBE-D3(B.J.), and PBE-LG corrections. Also, the equilibrium volume for (a and b) Cu, (c and d) Ag, and (e and f) Au. Here, we used the minimized structure from the DFT calculations (with or without vdW corrections) and the calculated vibrational energies to obtain the energy and pressure at the experimental temperature.

mixture of the ${ }^{3} \mathrm{~F}$ and ${ }^{3} \mathrm{P}$ states for the $(4 \mathrm{~s})^{2}(3 \mathrm{~d})^{8}$ configuration for Ni. This leads to a Ni atom energy too high by $0.535 \mathrm{eV}=$ $51.65 \mathrm{~kJ} / \mathrm{mol}$. We corrected this in the figure and tables (see Figure 6). To match the experimental cohesive energy, we used a negative $C_{6}$ to lower the overestimation from pure PBE.

$\mathrm{PBE}$ fails to provide consistent predictions of the cohesive energies of metals. In the metals investigated in this work, PBE underestimates the cohesive energies for metals like $\mathrm{Cu}, \mathrm{Ag}$, and $\mathrm{Au}$, but it overestimates the cohesive energy of $\mathrm{Ni}^{16}$ This inconsistency of PBE poses a great challenge to any postage correction with pure attraction. Advanced methods are expected to solve the above problem. The advantage of the effective method with embedding multiple effects into parameters is the minimal cost added to reproduce the known experimental results, which are important for applications, such as electrochemical reaction, that require accuracy within $0.1 \mathrm{eV}$.

van der Waals density functional, such as strongly constrained and appropriately normed (SCAN) with the nonlocal correlation part from the rVV10, has been demonstrated to be reliable in describing the interaction between organic and metals. Although the computational cost is about 10 times more than PBE-LG, SCAN+rvv10 provides reliable predictions when experimental results are not available. The advantage of PBE-LG lies in its high efficiency and accuracy for systems within (or close to) the training set.

Noble Gas Crystals. The standard D3 parameters for the noble gas atoms match accurately the two-body interactions for the diatomic system, including $1 / R^{8}$ terms and $1 / R^{6}$ terms as in eq 1 . However, this overestimates the binding in the crystal. The 

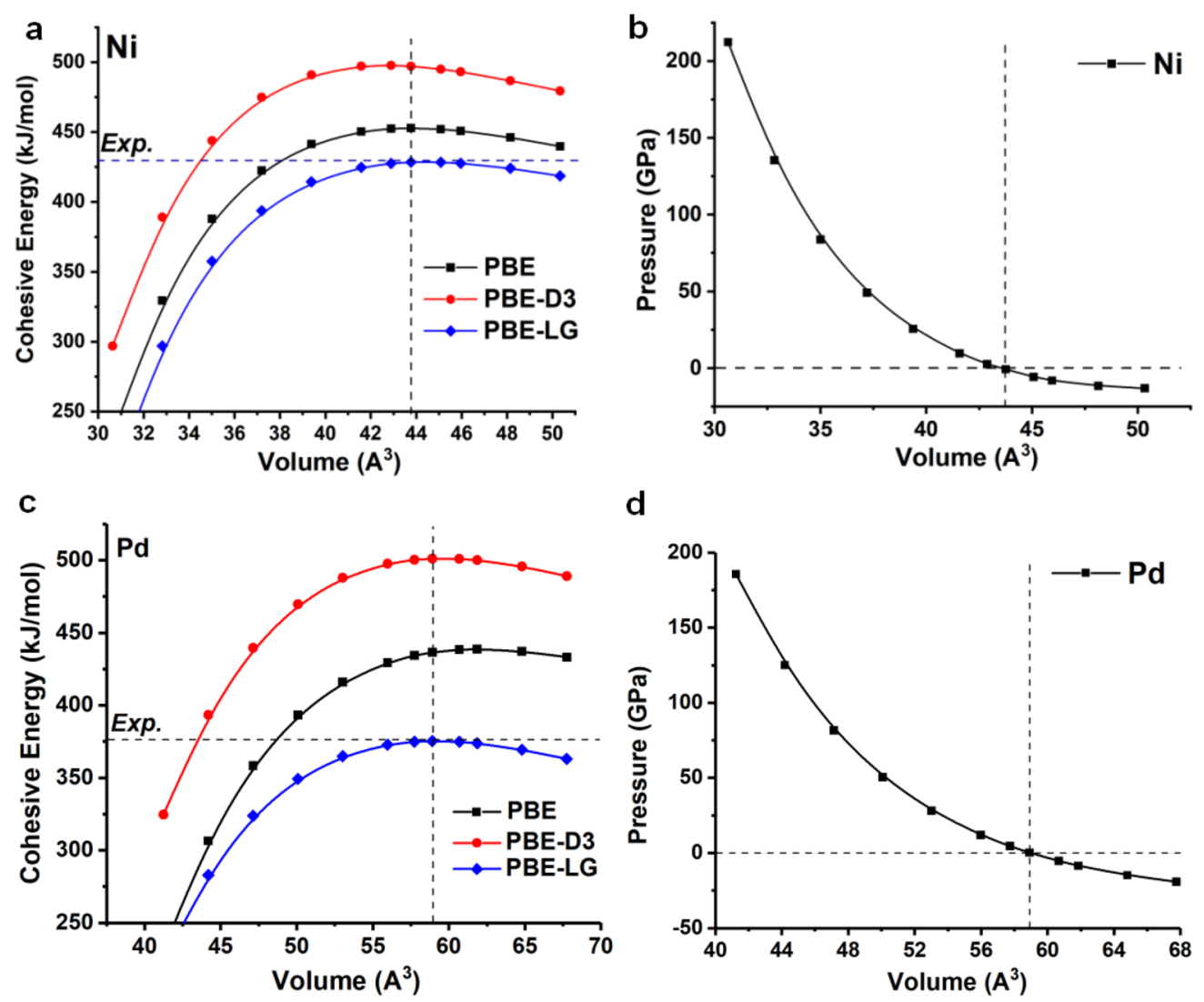

Figure 6. Comparison of cohesive energy of ( $a$ and $b$ ) Ni and ( $c$ and d) Pd between pure PBE, PBE-D3(B.J.), and PBE with LG corrections.
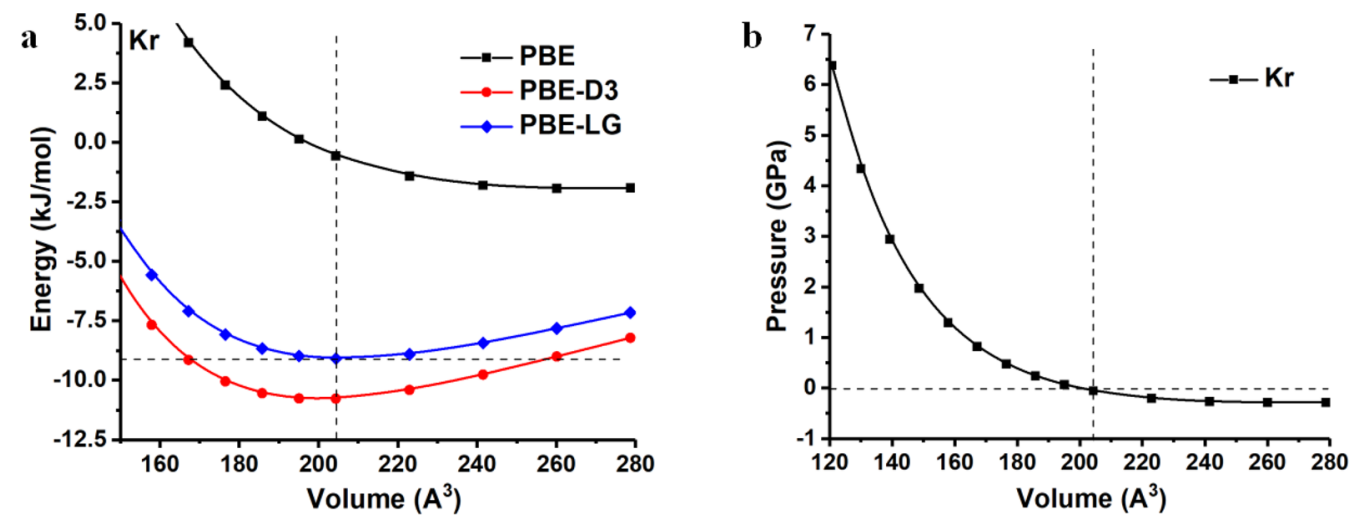

Figure 7. (a) Comparison of the calculated heat of vaporization of $\mathrm{Kr}$ crystal for various DFT methods compared to experiment. (b) Predicted and experimental equation of state for $\mathrm{Kr}$ crystal at $26 \mathrm{~K}$.

problem is that the third-order Axelrod-Teller dispersion interactions are also important in the crystal, which tends to reduce the net cohesive energy. We expect that such higherorder dispersion probably plays a similar role in all condensed systems, even if not crystalline, but including them in the simulations would be impractical.

Instead, we fit the two-body LG van der Waals to the experimental lattice parameters, the heat of vaporization, and equation of state. These two-body terms implicitly include the three-body Axelrod-Teller terms.

We will discuss the case of $\mathrm{Kr}$ in detail and summarize the results for the other noble gas crystals (Figure 7). Table 3 shows the PBE-D3 equation of state (EoS) for $\mathrm{Kr}$ and the EoS after correcting for zero-point energy (ZPE) and the temperature dependence of the free energy to $26 \mathrm{~K}$, the temperature for the experimental EoS. The predicted heat of vaporization at $26 \mathrm{~K}$, $\Delta H_{\text {vapor }}$ is compared with the experiment in Table 3, where we see that it is $120 \%$ too large.

The EoS for the other noble gases is included in the Supporting Information. The comparison of the $\Delta H_{\text {vapor }}$ for the other noble gases is in Table 3.

Trends in $v d W$ Parameters. We compare $C_{6}$ and $R_{0}$ for selected transition metal and corresponding Noble gases in Figure 8. It appears that the slope of $\log C_{6}$ vs $R_{\mathrm{vdw}}$ is steepest for $\mathrm{Ne}-\mathrm{Ar}$, which do not have core $\mathrm{d}$ electrons, less steep for $\mathrm{Ni}-\mathrm{Cu}-\mathrm{Kr}$ and $\mathrm{Pd}-\mathrm{Ag}-\mathrm{Xe}$ which have $\mathrm{d}$ core electrons but not $\mathrm{f}$, and even less steep for $\mathrm{Pt}-\mathrm{Au}-\mathrm{Rn}$ which also has $\mathrm{f}$ core electrons; perhaps these trends might be used to estimate corrections for other metals. 
Table 3. Heat of Vaporization of Rare Gas Face-CenteredCubic Crystal Systems (He, Ne, Ar, Kr, Xe, and Rn) Calculated Using PBE-LG Method, Compared with PBE-D3 (B.J.) Correction and Experiments ${ }^{a}$

\begin{tabular}{|c|c|c|c|c|c|}
\hline & \multicolumn{3}{|c|}{ heat of vaporization $\left(\mathrm{kJ} \cdot \mathrm{mol}^{-1}\right)$} & \multirow[b]{2}{*}{$R_{\mathrm{vdwLG}}(\AA)$} & \multirow[b]{2}{*}{$C_{6 \mathrm{LG}}(\mathrm{eV})$} \\
\hline & $E_{\mathrm{PBE}-\mathrm{D} 3}$ & $E_{\mathrm{PBE}-\mathrm{LG}}$ & exptl & & \\
\hline $\mathrm{He}$ & 0.63 & 0.09 & $0.08^{17}(T=0 \mathrm{~K})$ & 2.2239 & 0.7916 \\
\hline $\mathrm{Ne}$ & 2.40 & 1.91 & $1.92^{18}(T=24 \mathrm{~K})$ & 3.0534 & 10.2421 \\
\hline $\mathrm{Ar}$ & 7.16 & 7.66 & $7.73^{19}(T=24 \mathrm{~K})$ & 3.6418 & 236.6570 \\
\hline $\mathrm{Kr}$ & 10.78 & 9.08 & $9.02^{19}(T=26 \mathrm{~K})$ & 4.7630 & 815.7240 \\
\hline $\mathrm{Xe}$ & 19.64 & 14.93 & $14.94^{19}(T=24 \mathrm{~K})$ & 4.9990 & 2434.3320 \\
\hline $\mathrm{Rn}$ & 18.22 & 20.00 & $19.98^{18}(T=24 \mathrm{~K})$ & 6.8990 & 14001.5740 \\
\hline
\end{tabular}

${ }^{a}$ Here we have used the minimized structure from the DFT calculations (with vdW corrections), calculated vibrational energies, and used these to obtain the energy and pressure at the experimental temperatures.

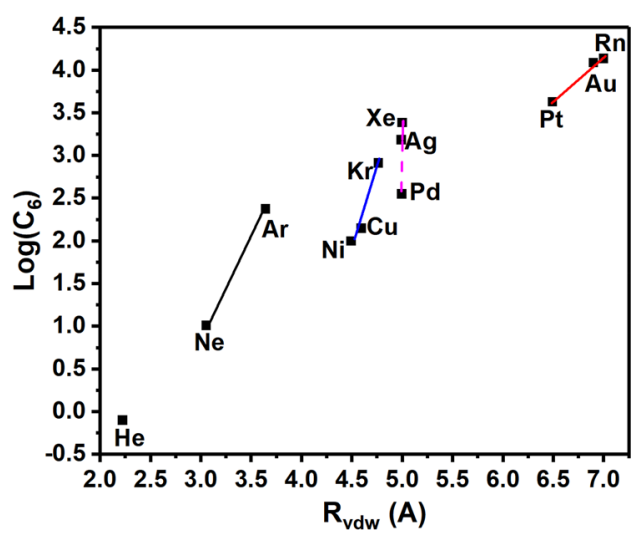

Figure 8. Plot of $R_{\mathrm{vdw}}$ versus $\log C_{6}$. The colored lines are linear fits for elements with similar core electron distributions. (The point here for $\mathrm{Pd}$ is only from predictions, along with the extended dashed line.)

The current strategy can be extended to heteroatomcontaining organic molecules, such as methanol. The adsorption energy of methanol on Pt predicted from PBE-LG is $-0.56 \mathrm{eV}$, which is almost the same as the experiment of $-0.59 \mathrm{eV}$. We will extend our current scheme to cover more metal-organic systems when experimental results are available.

In summary, we developed new vdW correction parameters for group 10 and 11 transition metals by fitting to experimental binding cohesive energy and for all six noble gases (i.e., $\mathrm{He}, \mathrm{Ne}$, $\mathrm{Ar}, \mathrm{Kr}, \mathrm{Xe}$, and $\mathrm{Rn}$ ) by fitting the heat of vaporization. This led to new parameters, $C_{6}$ and $R_{\mathrm{vdw}}$, derived for the simple low-gradient form. Using the newly fitted LG parameters of metals combined with previous $\mathrm{LG}$ parameters for $\mathrm{C}$ and $\mathrm{H}$, we find a good match to the experimental binding enthalpy for benzene and other hydrocarbons to $\mathrm{Pt}$ (111) surface. Also, we predict the adsorption energies of hydrocarbons on other metals using the same methodology. This simple LG correction should provide a good estimate for long-range London dispersion and hence for adsorption behaviors on surfaces.

\section{ASSOCIATED CONTENT}

\section{SI Supporting Information}

The Supporting Information is available free of charge at https://pubs.acs.org/doi/10.1021/acs.jpclett.0c03126.

Details of the computational methods; estimation of reaction enthalpy of benzene and other adsorbed species on the metal surface (PDF)
Details of the fitting of the new LG parameters (txt) Implementation of LG input for VASP (txt)

\section{AUTHOR INFORMATION}

\section{Corresponding Authors}

Tao Cheng - Institute of Functional Nano and Soft Materials (FUNSOM), Soochow University, Suzhou 215123, China; 이 orcid.org/0000-0003-4830-177X; Email: tcheng@ suda.edu.cn

William A. Goddard, III - Materials and Process Simulation Center, California Institute of Technology, Pasadena, California 91125, United States; 이이.org/0000-00030097-5716; Email: wag@caltech.edu

\section{Author}

Hao Yang - Institute of Functional Nano and Soft Materials (FUNSOM), Soochow University, Suzhou 215123, China; (1) orcid.org/0000-0002-8241-6231

Complete contact information is available at:

https://pubs.acs.org/10.1021/acs.jpclett.0c03126

\section{Notes}

The authors declare no competing financial interest.

\section{ACKNOWLEDGMENTS}

T.C. and H.Y. thank the National Natural Science Foundation of China (21975148), the Natural Science Foundation of Jiangsu Higher Education Institutions (SBK20190810), the Jiangsu Province High-Level Talents (JNHB-106), and the Priority Academic Program Development of Jiangsu Higher Education Institutions (PAPD) for financial support. H.Y. thanks China Postdoctoral Science Foundation (2019M660128) for financial support. This work was partly supported by the Collaborative Innovation Center of Suzhou Nano Science \& Technology. W.A.G. received support from the US National Science Foundation (CBET-1805022 and CBET-2005250).

\section{REFERENCES}

(1) Silbaugh, T. L.; Campbell, C. T. Energies of Formation Reactions Measured for Adsorbates on Late Transition Metal Surfaces. J. Phys. Chem. C 2016, 120, 25161-25172.

(2) Koch, W.; Holthausen, M. C. A Chemist's Guide to Density Functional Theory; Wiley-VCH: Weinheim, Germany, 2001.

(3) Zhang, Y.; Xu, X.; Goddard, W. A. Doubly Hybrid Density Functional for Accurate Descriptions of Nonbond Interactions, Thermochemistry, and Thermochemical Kinetics. Proc. Natl. Acad. Sci. U. S. A. 2009, 106, 4963-4968.

(4) Becke, A. D.; Johnson, E. R. A Unified Density-Functional Treatment of Dynamical, Nondynamical, and Dispersion Correlations. J. Chem. Phys. 2007, 127, 124108.

(5) von Lilienfeld, O. A.; Tavernelli, I.; Rothlisberger, U.; Sebastiani, D. Optimization of Effective Atom Centered Potentials for London Dispersion Forces in Density Functional Theory. Phys. Rev. Lett. 2004, 93, 153004.

(6) Grimme, S.; Antony, J.; Ehrlich, S.; Krieg, H. A Consistent and Accurate $\mathrm{Ab}$ Initio Parametrization of Density Functional Dispersion Correction (DFT-D) for the 94 Elements H-Pu. J. Chem. Phys. 2010, 132, 154104.

(7) Grimme, S.; Ehrlich, S.; Goerigk, L. Effect of the Damping Function in Dispersion Corrected Density Functional Theory. J. Comput. Chem. 2011, 32, 1456-1465.

(8) Naserifar, S.; Oppenheim, J. J.; Yang, H.; Zhou, T.; Zybin, S.; Rizk, M.; Goddard, W. A. Accurate Non-Bonded Potentials Based on Periodic Quantum Mechanics Calculations for Use in Molecular Simulations of Materials and Systems. J. Chem. Phys. 2019, 151, 154111. 
(9) Nørskov, J. K.; Bligaard, T.; Logadottir, A.; Kitchin, J. R.; Chen, J. G.; Pandelov, S.; Stimming, U. Trends in the Exchange Current for Hydrogen Evolution. J. Electrochem. Soc. 2005, 152, J23-J26.

(10) Liu, S.; White, M. G.; Liu, P. Mechanism of Oxygen Reduction Reaction on $\mathrm{Pt}(111)$ in Alkaline Solution: Importance of Chemisorbed Water on Surface. J. Phys. Chem. C 2016, 120, 15288-15298.

(11) Cave, E. R.; Montoya, J. H.; Kuhl, K. P.; Abram, D. N.; Hatsukade, T.; Shi, C.; Hahn, C.; Nørskov, J. K.; Jaramillo, T. F. Phys. Chem. Chem. Phys. 2017, 19, 15856-15863.

(12) Ye, Y.; Yang, H.; Qian, J.; Su, H.; Lee, K.-J.; Cheng, T.; Xiao, H.; Yano, J.; Goddard, W. A.; Crumlin, E. J. Nat. Commun. 2019, 10, 1875. (13) Hautier, G.; Ong, S. P.; Jain, A.; Moore, C. J.; Ceder, G. Accuracy of Density Functional Theory in Predicting Formation Energies of Ternary Oxides from Binary Oxides and Its Implication on Phase Stability. Phys. Rev. B: Condens. Matter Mater. Phys. 2012, 85, 155208.

(14) Carey, S. J.; Zhao, W.; Campbell, C. T. Energetics of Adsorbed Benzene on $\mathrm{Ni}(111)$ and $\mathrm{Pt}(111)$ by Calorimetry. Surf. Sci. 2018, 676, $9-16$.

(15) Charles, K. Introduction to Solid State Physics, 8th ed.; John Wiley \& Sons Inc.: Hoboken, NJ, 2005.

(16) Philipsen, P. H. T.; Baerends, E. J. Cohesive Energy of 3D Transition Metals: Density Functional Theory Atomic and Bulk calculations. Phys. Rev. B: Condens. Matter Mater. Phys. 1996, 54, 5326.

(17) Schumm, R. H.; Wagman, D. D.; Bailey, S.; Evans, W. H.; Parker, V. B. National Bureau of Standards (U.S.A.); Technical Notes, 270-1 to 270-8; 1973.

(18) McConville, G. T. New Values of Sublimation Energy L0 for Natural Neon and its Isotopes. J. Chem. Phys. 1974, 60, 4093.

(19) Schwalbe, L. A.; Crawford, R. K.; Chen, H. H.; Aziz, R. A. Thermodynamic Consistency of Vapor Pressure and Calorimetric Data for Argon, Krypton, and Xenon. J. Chem. Phys. 1977, 66, 4493-4502. 